\title{
Connections between Autism Spectrum Disorders (ASD) and Meares- Meares-Irlen Syndrome
}

\author{
Fabienne Giuliani' ${ }^{*}$ and Karin Schwarz ${ }^{2,3}$ \\ ${ }^{1}$ Psychiatry of Mental Development Section (Department of Psychiatry, CHUV, Suisse), Switzerland \\ ${ }^{2}$ Swiss Central Union for the Blind, Switzerland
}

${ }^{3}$ Optic Picto, Switzerland

\begin{abstract}
Aim: With this case study, we hypothesize that identical concomitant symptoms exist between ASDs and Meares-Irlen syndrome, determined by the level of visual stress.

Method: A questionnaire was given to subjectively identify the type and degree of symptoms associated with visual perception, and if these symptoms disrupted quality of life and learning process. This questionnaire was composed of questions on visual symptoms such as: symptoms linked to near and far visual distortion, binocular visual dysfunction and reading or writing errors experienced by the patient.

Results: This patient experienced a significant reduction in visual stress symptoms when wearing color-tinted lenses $(p<0.0001)$. With respect to our hypothesis, it appeared that treating the Meares-Irlen syndrome with the color-tinted lenses, (1) improved the patient's analysis of visual signals, and (2) reduced sensory hypersensitivity.

Conclusion: In this case study, it appeared that certain ASD deficits might have been caused by Meares-Irlen Syndrome, notably a change in the integration of sensory data. We wanted to highlight the lack of Meares-Irlen syndrome screening, which occurs because of a general unawareness of the syndrome. It is important to find ways to reduce this unawareness by developing the skill sets of different professionals in terms of adult ASD evaluations and consultations, by offering a scientific and holistic approach toward ASD individuals and by not excluding the possibility of an underlying Meares-Irlen syndrome.
\end{abstract}

Keywords: Autism spectrum disorders; Meares-Irlen syndrome

\section{Introduction}

Meares-Irlen syndrome was formerly known as the Syndrome of Scotopic Sensitivity [1]. It is a cluster of symptoms related to a neurological impairment in the processing of certain wave lengths contained in light [2] and it is considered a disorder of the magnocellular system causing a visual handicap as well as visual deformation and visual stress. In particular, it engenders difficulties in reading through the hyper-transmission of a specific light wave [3]. However, there is not yet a clear consensus regarding the etiology of this illness [1]. The symptoms of visual stress identified in the syndrome include perceptive distortion, asthenopia and headaches, diploplia, blurry vision, visual confusion and the implication of visual stress [4]. However, the problem is that Meares-Irlen syndrome symptoms are not specific, which is why a differential diagnosis is necessary in order to exclude other ophthalmological disorders [5]. The visual problems are alleviated by superimposing color [4]. Recently, a study by Kim et al. [1] detected important activation zones in fMRI in the middle temporal and upper left cortices while reading sentences using color-tinted lenses compared to reading sentences without any color-tinted lenses. This study, which was conducted on adult university students, showed a $21 \%$ improvement in reading speed when using color-tinted lenses, suggesting that these lenses provide a positive benefit.

\section{What are the possible links between ASDs and Meares-Irlen syndrome?}

Currently, no study has yet been published concerning ASDs and Meares-Irlen syndrome. For our part, we estimate that a great number of ASD individuals may be affected by Meares-Irlen syndrome even if the latter has not yet been diagnosed. This is our hypothesis because there is a concomitance of identical symptoms in ASDs and Irlen (sensory overload). We know that [6,7] the analysis of visual signals is done through three types of segregation: categorical (color, contrast, etc.), geometric (shape, length, edges, etc.) and dynamic (movement). This brain activity is guaranteed through segmentation of sensory input. It would appear that ASD individuals do not discriminate when processing sensory information $[8,9]$ and that their difficulty in reconstructing the complexity of the world comes from a problem in how they deal with different facets of unintegrated sensorimotor connections. In MearesIrlen syndrome, this problem of discrimination in the processing of sensory information comes from the functional morphology of sensorimotor connections which break the world down according to a repertory of predetermined properties. Visual signal analysis is disturbed (how to define a category of entering visual signals if these signals are badly defined). We are thus able to wonder about the pre-existence of Meares-Irlen syndrome in ASDs [10]. The sensory hypersensitivity of ASD individuals increases the salience of processed visual information [9] and we can also wonder if Meares-Irlen syndrome is not equally responsible. Autistic perception is slowed down because of difficulties integrating associative zones attributed to a problem of intra-cerebral connectivity $[11,12]$; Meares-Irlen syndrome may also play a role in this.

*Corresponding author: Fabienne Giuliani, SPDM, Site de Cery, 1008 Prilly, Switzerland, Tel: +4121 3142185; E-mail: fabienne.giuliani@chuv.ch

Received August 18, 2017; Accepted September 06, 2017; Published Septembe 13, 2017

Citation: Giuliani F, Schwarz K (2017) Connections between Autism Spectrum Disorders (ASD) and Meares-Meares-Irlen Syndrome. Autism Open Access 7: 216. doi:10.4172/2165-7890.1000216

Copyright: (c) 2017 Giuliani F, et al. This is an open-access article distributed unde the terms of the Creative Commons Attribution License, which permits unrestricted use, distribution, and reproduction in any medium, provided the original author and source are credited. 


\section{Materials and Method}

\section{Patient presentation}

Our patient was a 44 years old woman who was diagnosed with ASD according to the diagnostic criteria of the ICD-10 (F84.5) at the age of 39 by a psychiatrist. She was under regular outpatient treatment at the Section of Psychiatry of Mental Development (SPDM) at the University Hospital Center, Vaud (CHUV). She did not have any psychiatric co-morbidity. The diagnosis of Meares-Irlen syndrome was received at the age of 43 (H53.9 according to the ICD-10). A complete ophthalmological evaluation, including visual acuity, refractive errors, slit-lamp examination, tear break-up time, strabismus and retinal finding were conducted before receiving the Meares-Irlen syndrome diagnosis. The patient has given her consent for this publication.

\section{Test}

A paper version of a validated questionnaire with a large array of questions [1] was given to the patient. The questionnaire was designed to subjectively identify the type and degree of symptoms associated to visual perception, and whether these symptoms reduced quality of life and learning process. The questionnaire was composed of questions about visual symptoms such as: symptoms linked to near and far visual distortion, binocular visual dysfunction, and reading or writing errors experienced by the patient. This questionnaire was validated by previous studies on Meares-Irlen syndrome and visual discomfort [5].

\section{Data analysis}

We analyzed our patient's change in visual perception using closed questions (yes/no) as well as questionnaire elements which were graded using a four-point Likert scale of 1 (never) to 4 (always); an "I don't know" response type was marked as zero. A paired t-test was used and a difference was considered significant at $\mathrm{p}<0.05$. Questions focused on symptoms of visual stress and on sensitivity to light.

\section{Selection of tinted lenses}

The color-tinted lenses came from the Swiss Irlen Center and were made up of a variety of colored lenses. Each color provided four to five filters with differences in the intensity of shade. The patient first wore the lightest filters in each color group to be sure that she was comfortable with the color group. Within a selected color group, if the patient experienced a marked reduction in visual symptoms as well as an improvement of her visual perception, she was considered to be affected by Meares-Irlen syndrome [1,13].

\section{Results}

We followed this patient via outpatient consultation in the Service of Community Psychiatry dedicated to ASD adults. Early on, we documented clinically that she was bothered by the brightness of the lights. Her gaze was fixed and she appeared to be disturbed by colors, contrasts, shapes, and movements. She explained to us that she watches TV wearing sunglasses because she is very disturbed by the beams of light. Her visual disturbances have a negative impact on her cognitive capabilities. She explained to us that, "the data come in abruptly and she is unable to classify nor memorize them." She told us that she was unable to understand and follow her mathematics courses at school although she had been diagnosed as HIP (high intellectual promise). In that sense, this difficulty does not stem from a low intellectual capacity. She also told us that she had much trouble with reading and remembering what she has read.

\begin{tabular}{|c|c|}
\hline Prescription for glasses? & No \\
\hline Use of contact lenses? & No \\
\hline Is right-handed? & Yes \\
\hline Visit to ophtalmologist in the last 2 years? & Yes \\
\hline Have you received a diagnosis of a lazy eye? & No \\
\hline Has your vision ever been corrected? & No \\
\hline Have you received a diagnosis for dyslexia? & No \\
\hline Are you sensitive to light? & Yes \\
\hline
\end{tabular}

Table 1: Preliminary responses inspired by the Alanazi study [14].

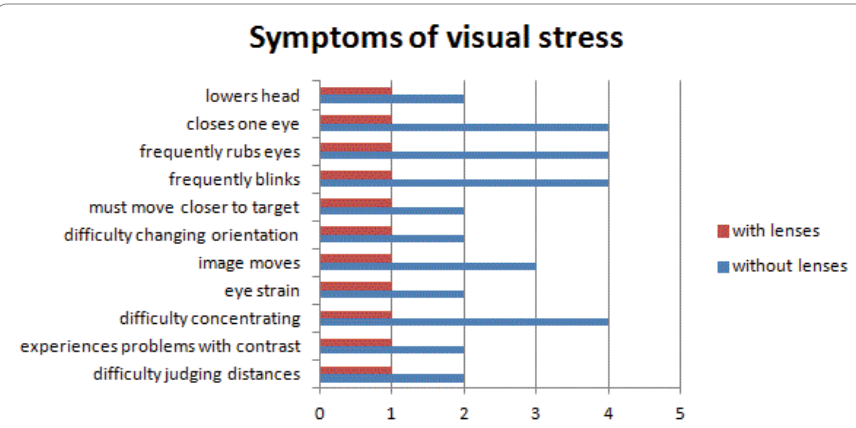

Figure 1: Analysis of the responses for symptoms of visual stress using a critical questionnaire (inspired by the Alanazi et al.'s study [14]) of visual perception with a Likert scale, with or without the use of color-tinted lenses.

The preliminary responses inspired by the Alanazi study [14] are listed in Table 1.

\section{Analysis of patient responses}

The patient's visual constraint scores are presented in Figure 1. These were the results obtained by the patient during visual perception tests without color-tinted lenses and then with the lenses. Scores range from $1=$ never; $2=$ a little, $3=\mathrm{a}$ lot; $4=$ always and $0=$ do not know. The average \pm standard deviation of the responses concerning the visual constraints before wearing the lenses was $2.8 \pm 0.9$ while the score with the lenses fell to $1 \pm 0$. The non-paired T-test on the visual constraints gave us a significant result, implying that there was a difference when the colored lenses were used $(t(10)=6.143, p<0.0001)$.

\section{Discussion}

We decided to evaluate the patient's visual stress using a subjective technique, meaning we used a self-assessment questionnaire. We found that the patient's symptoms of visual stress were significantly reduced when wearing the color-tinted lenses $(\mathrm{p}<0.0001)$. With respect to our hypothesis that this ASD patient was also subject to Meares-Irlen syndrome, it appeared indeed that the syndrome was alleviated through the use of color-tinted lenses and gave the patient (1) a better analysis of visual signals, (2) a decrease in sensory hypersensitivity.

Nevertheless, the most significant element for us was the patient's testimony. After the color-tinted lenses were put in place, the patient said: «I can read and work for hours without moving, without any pain or eye pain. My attention span and ability to concentrate are doubled. This makes a huge difference to me. The lenses make it so that the contrast is better for reading letters and the pages are less glaring and letters don't "move" (it makes me laugh to write that, because a psychiatrist had told me a long time ago: visual hallucinations! A problem with Ziprexa!) My eyes no longer hurt at all-and it's been more than 2 hours up that I've been wearing them this morning and everything is fine and nothing hurts at all. I was able to do my work, my reading and writing normally and 
without any pain, without anything. With the bright light right now (the days are longer and the reflection from the snow) I wore them yesterday at noon to make my lunch and to eat and it was just really cool, too - I could do all of that without experiencing any pain in my eyes or getting teary eyes and without putting on my sunglasses (something I had to do before). Yesterday evening I played on my game console and I could do this for more than an hour (before I could play about $20 \mathrm{~min}$ ) without my eyes hurting. I wanted to test playing on the television - something which was impossible before - and I could play on the big screen for $30 \mathrm{~min}$ - not more than that because it's no longer really my thing, it's too big and like a movie on the television; the secession of images is often too quick for me. In my writing work with the pages I still started inverting the numbers and letters at the end of $2 \mathrm{~h}$ of this kind of activity - there again - this is really more a lack of attention on my part, I think, but at least I could see it when I was rereading. As planned yesterday afternoon I wore my normal sunglasses to go out and there I experienced the usual things: my eyes hurt, everything was less contrasted, it was interesting to see. It seems to me then that these color-tinted lenses change a lot of things."

\section{Study Limitations}

There are several limitations to this study. First, this is just the presentation of a single case study and because of this the protocol must be replicated and used on a larger number of ASD cases in future studies. Secondly, there are no current studies of Meares-Irlen syndrome on a population diagnosed with autism spectrum disorders. Thirdly, the controversy concerning the diagnostic criteria for Meares-Irlen syndrome is obviously an inherent limitation. Finally, this study was a pilot study and did not involve a matched control situation.

\section{Conclusion}

In conclusion, our ASD patient significantly reduced her symptoms of visual stress by wearing color-tinted lenses. On the one hand, this improved her quality of life (to be able to go out, to enjoy views of landscapes without any visual disturbances) and on the other hand, this had an impact on her quality of learning. For this article, we have suggested an understanding of certain deficits of ASD as a consequence of Meares-Irlen syndrome, especially a change in the integration of sensory data. It is important to find the means to reduce this unawareness in any possible way, notably by developing the skill sets of different professionals and particularly those working in psychiatry specialized in the field of evaluations and consultations for ASD adults, by offering a scientific and holistic approach for ASD individuals and by not excluding the possibility of an underlying Meares-Irlen syndrome.

\section{Conflict of Interest}

No potential conflict of interest relevant to this article was reported.

\section{References}

1. Kim JH, Seo HJ, Ha SG, Kim SH (2015) Functional magnetic resonance imaging findings in Meares-Irlen syndrome: A pilot sudy. Korean J Ophthalmol KJO 29: 121-125.

2. Barbolini G, Lazzerini A, Pini LA, Steiner F, Del Vecchio G, et al. (2009) Malfunctioning cones and remedial tinted filters. Ophta 2: 101-107.

3. Chase C, Ashourzadeh A, Kelly C, Monfette S, Kinsey K (2003) Can the magnocellular pathway read? Evidence from studies of color. Vision Res 43 $1211-1222$

4. Kruk R, Sumbler K, Willows D (2008) Visual processing characteristics of children with Meares-Irlen syndrome. Ophthalmic Physiol Opt 28: 35-46.

5. Evans BJ (2005) The need for optometric investigation in suspected MearesIrlen syndrome or visual stress. Ophthalmic Physiol Opt 25: 363-370.

6. Giuliani F, D'Armi N (2016) Particularities of visual scanning in static vs. dynamic situations for Asperger's subjects: New advance in ASDs. Austin J Autism Relat Disabil 2: 1028-1032.

7. Giuliani F, Schenk F (2015) Vision, spatial cognition and intellectual disabilities Res Dev Disabil 37: 202-208.

8. Giuliani F, El Korh P (2015) Psychotherapy for adults with autistic spectrum disorders. Adv Techn Biol Med 4: 158.

9. Gomez JC (2009) Embodying meaning: Insights from primates, autism and Brentano. Neural Networks 22: 190-196.

10. Safra D, Steiner F (2008) Autismus und Sehen. Ophta 4.

11. Baron-Cohen S, Ashwin E, Ashwin C, Tavassoli T, Chakrabarti B (2009) Talent in autism: Hyper-systemizing, hyper-attention to detail and sensory hypersensitivity. Philos Trans R Soc Lond B 364: 1377-1383.

12. Mottron L, Dawson M, Soulieres I (2009) Enhanced perception in savan syndrome: Patterns, structure and creativity. Philos Trans R Soc London B 364: 1385-1391.

13. Park SH, Kim SH, Cho YA, Joo CK (2012) The effect of colored filters in patients with Meares-Irlen syndrome. J Korean Ophtalmol Soc 53: 452-459.

14. Alanazi MA, Alanazi SA, Osuagwu UL (2016) Evaluation of visual stress symptoms in age-matched dyslexic, Meares-Irlen syndrome and normal adults. Int J Ophthalmol 9: 617-624 